\title{
Improving the Flexural Strength Test of Brushite Cement
}

\author{
Sahin Altundal ${ }^{1, a^{*}}$, Karlis Agris Gross ${ }^{2, b}$, Caroline Ohman ${ }^{3, c}$, Hakan Engqvist ${ }^{4, d}$ \\ ${ }^{1}$ Institute of Biomaterials and Biomechanics, Riga Technical University, Latvia \\ ${ }^{2}$ Applied Materials Science, Department of Engineering Sciences, Uppsala University, Sweden \\ âsahinaltundal@gmail.com, 'Karlis-Agris.Gross@rtu.lv, 'caroline.ohman@angstrom.uu.se, \\ dhakan.engqvist@angstrom.uu.se
}

\begin{abstract}
Keywords: Error possibilities in bending test, Weibull effective surface, Weibull effective volume, bearing support design, effect of cement injection.
\end{abstract}

\begin{abstract}
In the investigation of mechanical properties, calcium phosphate cements exhibit large sample-to-sample deviation due to its porous nature, possibility of un-homogenous distribution and small specimen size. This situation generates difficulties for obtaining accurate results and creates an obstacle for testing different composition where only a small batch size is available. In this respect, specimen shape, whether being injected, porosity ratio, surface quality, bearing support design have significant matter on variability in terms of three- and four-point bending test. Therefore, different methods have been studied to reduce variability with a simpler material preparation than common methods on injected and moulded cement. The entire comparison is made with the consideration of three- and four-point bending testing, the eccentric loading error calculation with engineering calculation software "Mathcad 15", porosity measurement with Archimedes method, microstructure investigation on Scanning Electron Microscope (SEM), macroporosity distribution measurement by Micro CT Scanner.
\end{abstract}

\section{Introduction}

Since the mechanical investigation took a significant part to improve calcium phosphate cement in the last half of $20^{\text {th }}$ century, to obtain comparable results in flexural strength testing, standardization has been needed. However, difficulties in the specimen preparation due to brittle nature and possessing low mechanical strength of calcium phosphate cements generate some problems such as breakage during the specimen preparation, dimension instability and in addition producing cracks in polishing stage. Therefore, a more stable cross section such as circular shape than rectangular cross section might be useful in flexural strength testing. Moreover, there is no need for surface treatment in the preparation of circular specimens and they are beneficial to avoid edge crack which may take place from surface preparation process or surface macro-porosities [12]. As well as all these superior advantages, cylindrical specimens are inefficient for either volume- or surface-flaws comparing traditional rectangular specimens according to Weibull analysis [11]. However, circular specimen shape possesses some other disadvantages such as poor contact between bearing support and specimen herewith low force distribution and rotation on the flexural testing fixture. Thus, other bearing support designs must be considered against these drawbacks.

In the general assumptions of the testing of porous materials, four-point bending testing set-up is preferred as a more suitable option than three-point bending testing set-up due to the four point flexure fixture produces peak stresses along an extended region of the specimen surface [1]. Because, exposing a larger area of the specimen is possible with more potential for defects and flaws. Hereby, the effect of sensitivity of testing equipment in a small misalignment of the testing fixture in both four- and three-point bending testing has been investigated for such small sample batches.

Porosity distributions and proportions are the other effective parameters on mechanical properties. Hence, the effect of the injection of brushite cement is investigated to porosity discontinuities which might induce variability in the testing. While Archimedes principle is used for overall porosity measurement, micro $\mathrm{CT}$ analysis is used for macro-porosity $(>7.88 \mu \mathrm{m})$ distribution, and proportion measurement. 


\section{Materials and methods}

Sample preparation. The brushite cement was obtained from a mixture of monocalcium phosphate monohydrate (MCPM, $>97 \%$, Alfa Aesar,Germany) and $\beta$-tricalciumphosphate $(\beta$-TCP, $>96 \%$, Sigma-Aldrich,Germany). The average particle size of the $\beta$-TCP and MCPM was used as $13.6( \pm 0.10) \mu \mathrm{m}$, and $<75 \mu \mathrm{m}$ respectively. Then, MCPM and $\beta$-TCP were mixed with 45:55 molar ratio, adding $1 \mathrm{wt} . \%$ pyrophosphate, during at least half an hour in a turbula machine (System SCHATZ, Willy A. Bachofen AG machinen fabrik Switzerland) [2,3].

The liquid phase was prepared as a citric acid solution with a 0.5 molar concentration was prepared by dissolving $9.606 \mathrm{~g}$ of citric acid powder in $100 \mathrm{ml}$ of deionized water [2,3].

To prepare the brushite cement, the liquid and the powder phases were stirred at a L/P ratio of 0.22 , first via a Cap-Vibrator machine (for a few seconds) and then via a Vortex-Genie 2 for $2 \times 30$ seconds in $50 \mathrm{ml}$ falcon tube.

Silicon rubber moulds were prepared with a $4 \mathrm{~mm}$ diameter and $40 \mathrm{~mm}$ height cavity. After the liquid phase contacted the powder phase, the cement was moulded with a spatula and a $10 \mathrm{ml}$ syringe, with a $1.90 \mathrm{~mm}$ outlet diameter, individually in 5 minutes. Subsequently the specimens were taken in containers and were immersed in $60 \mathrm{ml}$ of phosphate buffered saline (PBS, $0.01 \mathrm{M}$ phosphate buffer, $0.0027 \mathrm{M}$ potassium chloride and $0.137 \mathrm{M}$ sodium chloride, $\mathrm{pH}$ 7.4, SigmaAldrich, Germany) at $37^{\circ}$ for $24 \mathrm{~h}$.

Mechanical testing. The flexural strength was measured using a universal materials testing machine (Shimadzu AGS-X, Japan) at a cross-head speed of $1 \mathrm{~mm} / \mathrm{min}$. At least 12 specimens were tested from each batch. All the measurements are made with wet samples (soaked in isopropanol). $[5,6]$. Three different design of bearing support were used in bending tests (Fig. 2).

For all the three- and four-point bending tests, cylindrical inner supports, that were $4 \mathrm{~mm}$ in diameter, were used for flexural strength measurement. Type-1, Type- 2 and Type- 3 bearing support fixtures are illustrated in Fig. 2. For Type-1 bearing support design, likewise, $4 \mathrm{~mm}$ diameter cylindrical outer supports were used. In Type- 2 design of fixtures, the curvature was $4 \mathrm{~mm}$ in diameter, $1 \mathrm{~mm}$ in depth and the diameter of the cylinders were specified as $5 \mathrm{~mm}$ for outer supports. In Type-3 design, the lateral diameter of the bearing support and the curvature diameter were designated as $4 \mathrm{~mm}$ and $22 \mathrm{~mm}$ respectively. The outer support span was chosen as $30 \mathrm{~mm}$ in both three- and four point bending testing fixtures and for the four-point bending tests the inner support span was $10 \mathrm{~mm}[10]$.

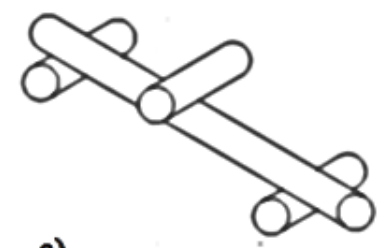

a)
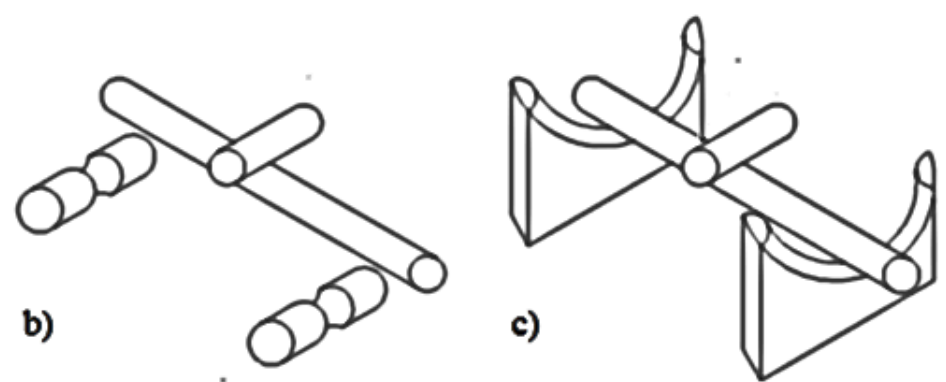

Fig. 2 - a) Type-1, b) Type-2 and c) Type-3 designs of bearing supports, used with three-point bending test fixture.

Flexural strength of a cylindrical rod using three-point bending [4]:

$$
\sigma_{f}=\frac{8 F L}{\pi D^{3}}
$$

Equation 2 gives flexural strength of a cylindrical rod using four-point bending fixture with 10 $\mathrm{mm}$ of length span of inner support [4-5].

$$
\sigma_{f}=\frac{16 F L}{3 \pi D^{3}}
$$


where $\sigma_{f}$ is the flexural strength (MPa), $F$ is the applied load $(\mathrm{N}), L$ is the length of span between outer supports $(\mathrm{mm}), D$ is the mean diameter of the sample $(\mathrm{mm})$.

\section{Porosity}

Overall porosity measurement. Archimedes principle was applied in order to determine the volume of pores in the samples. The solvent exchange method is used to obtain wet samples after the porosity measurement. After setting in PBS solution for $24 \mathrm{~h}$, the wet samples for the mechanical testing were weighed, firstly in air to obtain the mass of wet samples and then while suspended in a fluid (weight of wet sample in water). After the first two weightings, subsequently, the samples were stored in $\mathrm{C}_{3} \mathrm{H}_{8} \mathrm{O}$ (isopropanol) for $24 \mathrm{~h}$ and the weight reduction was monitored [7]. The temperature of the room was observed at $21^{\circ} \mathrm{C}$.

The porosity is calculated according to the equation 4 [7], where $\mathrm{V}_{\mathrm{a}}$ is the apparent volume [3], $\mathrm{m}_{\text {air }}$ is the weight of wet sample, $\mathrm{m}_{\text {water }}$ is weight of the sample under the water, $\mathrm{m}_{\text {alcohol }}$ is the weight after complete exchange, $\rho_{\text {water }}$ is the density of water, and $\rho_{\text {alcohol }}$ is the density of the alcohol used.

$$
\begin{aligned}
& \Phi(\%)=\left(\frac{\left(\mathrm{m}_{\text {air }}-\mathrm{m}_{\text {alcohol }}\right) /\left(\rho_{\text {water }}-\rho_{\text {alcohol }}\right)}{\mathrm{V}_{\mathrm{a}}}\right) \cdot 100 \\
& \mathrm{~V}_{\mathrm{a}}=\left(\mathrm{m}_{\mathrm{air}} / \rho_{\text {water }}\right)-\left(\mathrm{m}_{\text {water }} / \rho_{\text {water }}\right)
\end{aligned}
$$

Macro-porosity measurement. Volume porosity distribution was detected by using a tomographic measurement device (Micro-CT Skyscan 1172, Brussels, Belgium). The resolution is used as 7.88 $\mu \mathrm{m}$ pixel size. For the testing, 6 cylindrical bending test specimens were prepared and dried in air for at least $48 \mathrm{~h}$.

\section{Microstructure}

The microstructure of polished cross-sections was analyzed using scanning electron microscopy (SEM, Hitachi TM-1000 TableTop Microscope, Krefeld, Germany). The samples were dried in air for at least 1 week at $21^{\circ} \mathrm{C}$. The samples were roughly polished with grit $800 \mathrm{SiC}$ abrasive paper.

\section{Results and discussion}

Mechanical testing. From the testing samples, only 10 significant results were chosen from each batch of samples. Since the volume under stress in four-point bending test is larger than three-point bending test, the four-point bending set-up exhibited lower flexural strength in the measurements as expected considering Weibull statistics [8,9]. All the three- and four-point bending test batches demonstrated similar average mechanical strength among each other.

In contrast to the general assumption, three-point bending testing set-ups have demonstrated lower standard deviation. This phenomena is also related to higher error probability of four-point bending testing in such small samples as well as arbitrary function. Fig. 4-a illustrates two possible errors: the poor articulation and the eccentric loading. Poor articulation error is related to wrong settlement of the testing fixture and instability in the sample dimensions. This problem is avoided with adapting inner support fixture with a spring mechanism which is made to balance two inner supports so that, they will contact on the sample surface together during the loading with a slightly different force distribution. The eccentric loading error occurs when the inner supports are not settled on the center of two outer supports. Loading points and misalignment are shown in Fig. 4-b. Equation 6-8 gives the error percentages depending on misalignment of the supports [5].

For four-point bending test fixture:

$$
\varepsilon=\frac{-2\left(\frac{e}{L}\right)+\left(\frac{e}{a}\right)+2\left(\frac{e}{a}\right)\left(\frac{e}{L}\right)}{1+2\left(\frac{e}{L}\right)-\left(\frac{e}{a}\right)-2\left(\frac{e}{a}\right)\left(\frac{e}{L}\right)} \times 100 \text { Point } 2
$$




$$
\varepsilon=\frac{+2\left(\frac{e}{L}\right)-\left(\frac{e}{a}\right)+2\left(\frac{e}{a}\right)\left(\frac{e}{L}\right)}{1-2\left(\frac{e}{L}\right)+\left(\frac{e}{a}\right)-2\left(\frac{e}{a}\right)\left(\frac{e}{L}\right)} \times 100 \text { Point } 3
$$

For three-point bending test fixture:

$$
\varepsilon=\frac{+4\left(\frac{e}{L}\right)^{2}}{1-4\left(\frac{e}{L}\right)^{2}} \times 100
$$

where $a$ is the length of span between inner supports $(\mathrm{mm}), L$ is the length of span between outer supports $(\mathrm{mm}), e$ is length of misalignment $[5,6]$.

In Fig. 4-a, change in error percentage up to $2 \mathrm{~mm}$ of misalignment is demonstrated for threeand four-point bending measurements for specified dimension of testing equipment. For the four point bending set-up, the greater error occurs directly at point 2, but the greatest stress, which is more likely to cause fracture, occurs at point 3. For $1 \mathrm{~mm}$ of misalignment, while three-point bending set-up is giving $0.45 \%$ error, four-point bending set-up gives $4.17 \%$ error at point 2 and $2.60 \%$ error on point 3 .

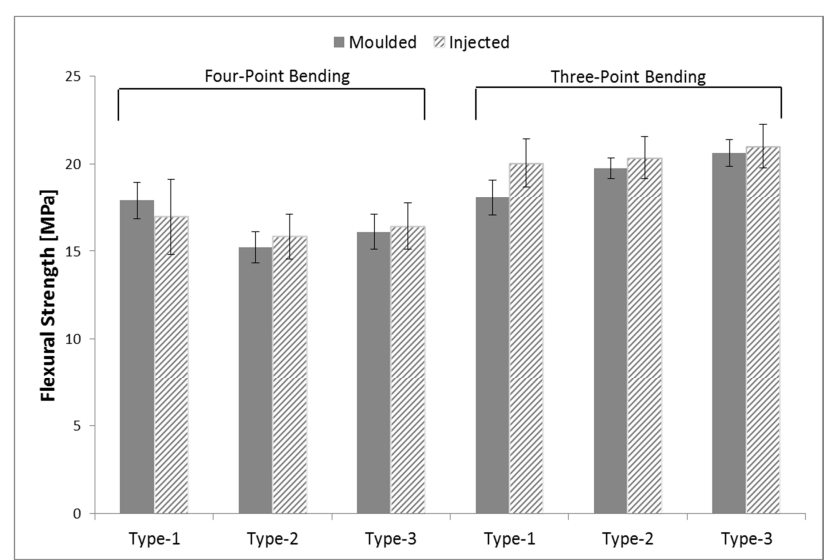

Fig. 3 - Flexural strength testing results with specimens of moulded and injected cement, using different testing fixtures.

Among three bearing support designs, there is not a sharp difference in terms of mechanical strength depending on the bearing supports (Fig. 3). On the other hand, they have exhibited different deviations in order. In both three- and four-point bending fixtures, Type- 1 and Type- 2 have demonstrated the largest and the smallest standard deviation respectively among all three designs of bearing support. There are two possible effective phenomena relevant to the effect of bearing support design: the static force distribution on the bearing supports and the rotation of the specimens. The possible rotation and the small motion of the specimens were reduced by adapting the diameter of specimens to the diameter of the bearing supports. Thereby, Type- 2 represents the lowest possibility of the rotation or motion due to possessing the highest contact to the specimen with the same curvature diameter as the specimen diameter and the opposite for Type-1 fixture design.

Injected cement in general showed slightly greater flexural strengths than the cement that was moulded with spatula. In terms of variability, it similarly showed higher standard deviation values. This circumstance is mainly related to the distribution and settlement of the porosity. 
a)
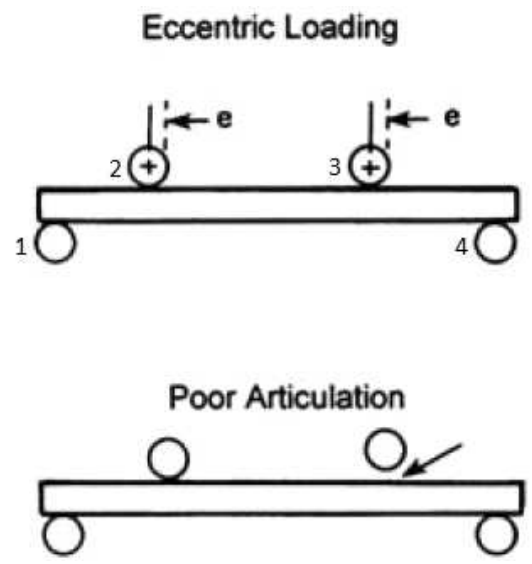

b)

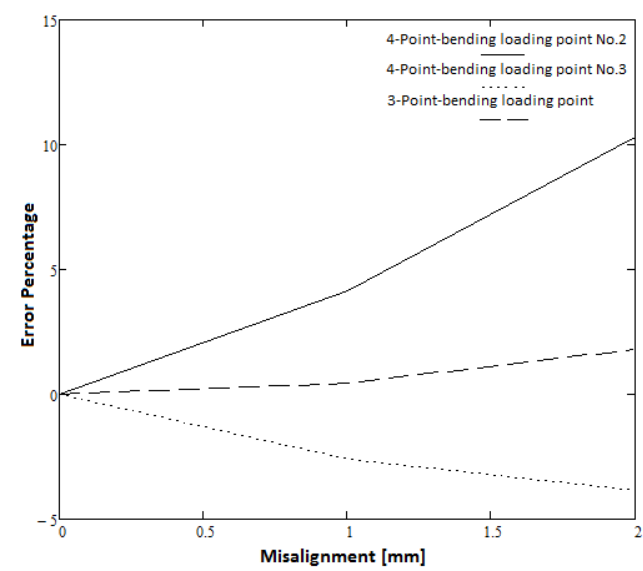

Fig. 4 - a) Two common errors in flexural strength tests: eccentric loading and poor articulation. b) Error percentages depending on misalignment in eccentric loading. Error percentages on point No. 2 and No.3 in four-point bending and three point bending inner supports.

Porosity measurement. The entire porosity proportion is calculated by Archimedes principle. For the porosity measurement of Archimedes principle, the same samples, which were used in mechanical testing, were used. The calculated average porosity proportion for moulded cement is 21.27 \pm 4.28 and for injected cement is $19.93 \pm 3.21$.

Macro-porosity distribution of the cylindrical samples that are prepared for micro CT is presented in Fig. 5-a. Injected cement reaches highest and lowest picks on the lower mid-range of pore size cement in general. The micro CT analysis shows that injected cement may present different pore size distribution.

The macro-porosity percentage is determined as $1.14 \pm 0.18$ for moulded cement and $1.10 \pm 0.30$ for injected cement by micro CT analysis. The average macro-porosity difference of the samples between moulded and injected cement is negligible. On the other hand, injected cement exhibits quite larger sample-to-sample deviation in terms of macro-porosity in the average volume percentage.

a)

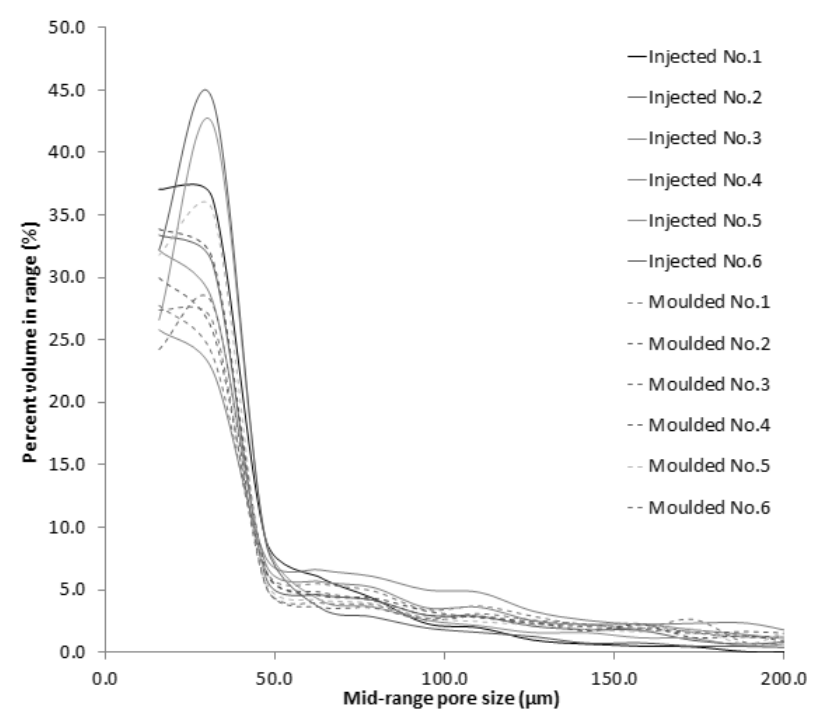

b)

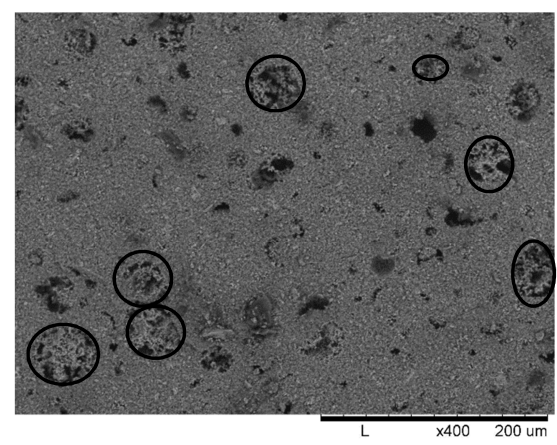

c)

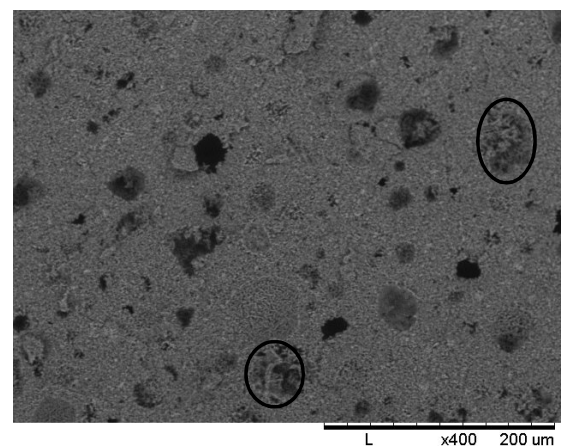

Fig. 5 - Porosity from a) micro-CT, b) moulded and c) injected cements, showing an average pore size of $35 \mu \mathrm{m}$. The moulded cement displays a contribution from larger pores. 


\section{Conclusion}

For the small sample lengths, 4-point bending test fixture shows a higher error probability than 3-point bending test fixture.

Among three different bearing support designs, Type-2 has demonstrated the lowest variability due to its prevention of motion towards a third direction as well as its considerable force distribution.

Injected cement has relatively lower porosity levels in terms of both entire and macro-porosity. This might be the evidence of the small difference on the flexural strength. On the other hand, injected cement exhibited higher sample-to-sample deviation in contrast to possessing better distribution of porosity. This circumstance is explained with high sample-to-sample deviation of the macro-porosity amount.

\section{Acknowledgements}

The authors acknowledge support from "Biomaterials for Bone Replacement" project (00845/2011) from Swedish Institute and Uppsala University, Biomaterials Institute.

\section{Reference}

[1] I.D. Baere, W.V. Paepegem, J. Degrieck, Design of a three- and four-point bending setup for fatigue testing of fibre-reinforced thermoplastics, FirW Phd Symposium (2005).

[2] Engstrand, J. Cecilia, P. Engqvist, H., The effect of composition on mechanical properties of brushite cements, Journal of the mechanical behavior of biomedical materials, 29 (2014) 8190.

[3] C. Robo, Study of calcium phosphate cements, Industrial memorial report, ETSEiB/BIBITE, (2013).

[4] D.A. Stewardson, A.C. Shortall, P.M. Marquis, P.J. Lumley, The flexural properties of endodontic post materials, 26 (2010) 730-736.

[5] G.D. Quinn, B.T. Sparenberg, P. Koshy, L.K. Ives, S. Jahanmir, D. D. Arola, Flexural Strength of Ceramic and Glass Rods, Journal of Testing and Evaluation, 37 (2009) 1-22.

[6] ASTM Standard C158-95, Standard Test Methods of Flexure Testing of Glass (Determination of the Modulus of Rupture), 15.02 (1998).

[7] S. Lamberet, Durability of ternary binders based on Portland cement, calcium aluminate cement and calcium sulfate: École polytechnique fédérale de Lausanne, Institute of Materials, Lyon, France, 2005.

[8] M.H. Dielhof, L.J.M.G. Dortmans, G. de With, Fractography of Borosilicate Glass Tested in Three- and Four-Point Bending, Journal of the European Ceramic Society, 12 (1993) 215-220.

[9] G.D. Quinn, J.B. Quinn, A practical and systematic review of Weibull statistics for reporting strengths of dental materials, Dental Materials, 26 (2009) 135-147.

[10] S. Wakayama, T. Kawakami, S. Kobayashi, M. Aizawa, A. Nozue, Acoustic Emission From Acoustic Emission from Micro-Fracture Processes of Bio-Ceramics in Simulated Body Environment, Journal of Acoustic Emission, 21 (2004) 149-157.

[11] G. D. Quinn, Weibull Effective Volumes and Surfaces for Cylindrical Rods Loaded in Flexure, Journal of the American Ceramic Society, 86 (2003) 475-479.

[12] P.J. Bates, C.Y. Wang, The Effect of Sample Preparation on The Flexural Strength of Reinforced Nylon 66, Polymer Engineering and Science, 43 (2003) 759-773. 
Bioceramics Volume 26

10.4028/www.scientific.net/KEM.631

Improving the Flexural Strength Test of Brushite Cement

10.4028/www.scientific.net/KEM.631.67 